\title{
CORRECTIONS
}

\section{High INR on warfarin}

In this Practice article (BMJ 2015;350:h1282, doi:10.1136/bmj. h1282), the third paragraph in the "What you should cover" section was amended on 10 June 2015 to conform with guidance from the National Institute for Health and Care Excellence on head injury. This recommends a computed tomography head scan within eight hours for all patients with a head injury while taking warfarin, regardless of any other risk factors; specific clinical features also determine the urgency of this scan.

Cite this as: BMJ 2015;350:h3200

๑ BMJ Publishing Group Ltd 2015 